\title{
Sobre algunas relaciones entre la teoría de la mentalización y el psicoanálisis
}

\author{
Gustavo Lanza Castelli ${ }^{1}$ \\ AIEDEM
}

\begin{abstract}
El trabajo propone una comparación y una articulación entre la teoría de la mentalización y el psicoanálisis, partiendo de cómo cada uno de ellos teoriza sobre la situación planteada por Winnicott en la que el niño se encuentra a sí mismo en el rostro de la madre, la cual funciona como espejo de aquél. En primer término el trabajo reseña el modo en que la teoría de la mentalización conceptualiza dicha situación a partir de su punto de vista específico, consistente en su interés por dilucidar los orígenes y naturaleza de la capacidad del niño para identificar y atribuir estados mentales, tanto a sí mismo como a los demás. Tras ello, se reseña el punto de vista psicoanalítico, centrado en comprender la constitución del narcisismo y la identidad, a partir de las identificaciones primarias y de la investidura idealizadora que la madre hace recaer sobre su hijo. Tras llevar a cabo una comparación entre ambas perspectivas se las aplica en una viñeta clínica con la intención de ilustrar la comprensión que cada una de ellas permite, así como el abordaje clínico que propone. Se subrayan las diferencias, como así también las complementariedades entre ambos enfoques.
\end{abstract}

Palabras clave: mentalización - psicoanálisis - rostro materno como espejo

The paper proposes a comparison and an articulation between the theory of mentalization and psychoanalysis, starting from how each of them theorizes about the situation posed by Winnicott in which the child finds himself in the mother's face, who functions as a mirror of the child. Firstly, the paper reviews the way in which the theory of mentalization conceptualises this situation from its specific point of view, consisting of its interest in elucidating the origins and nature of the child's capacity to identify and attribute mental states, both to himself and to others. After that, the psychoanalytic point of view is reviewed, focused on understanding the constitution of narcissism and identity, from the primary identifications and the idealizing investment that the mother makes on her son. After a comparison between both perspectives, they are applied in a clinical vignette with the intention of illustrating the understanding that each one allows, as well as the clinical approach that they propose. The differences, as well as the complementarities between the two approaches, are highlighted. Key Words: mentalization - psychoanalysis - mother's face as mirror

English Title: On some relations between the theory of mentalization and psychoanalysis

\section{Cita bibliográfica / Reference citation:}

Laza Castelli, G. (2021). Sobre algunas relaciones entre la teoría de la mentalización y el psicoanálisis. Clínica e Investigación Relacional, 15 (1): 117-137. [ISSN 1988-2939] [Recuperado de www.ceir.info ] DOI: 10.21110/19882939.2021.150106

\footnotetext{
${ }^{1}$ Psicoanalista. Presidente de la Asociación Internacional para el Estudio y Desarrollo de la Mentalización (AIEDEM). Ha sido profesor en distintas universidades y postgrados en Argentina. Actualmente es profesor en el Grupo de Psicoterapia Analítica de Bilbao. Director de Mentalización. Revista de Psicoanálisis y Psicoterapia. Es autor de más de 70 trabajos, publicados en revistas nacionales y extranjeras, sobre psicoanálisis, psicopatología, escritura y psicoterapia, mentalización y psicoterapia. Ha diseñado dos pruebas para evaluar la mentalización Correo electrónico: gustavo.lanza.castelli@gmail.com
} 
La teoría de la mentalización, presentada originariamente por Fonagy (1991), Fonagy y Target (1996), Target y Fonagy (1996), Fonagy et al. (1998) y la práctica terapéutica que de ella deriva, la terapia basada en la mentalización (MBT por sus siglas en inglés) se han extendido de manera exponencial en los últimos 20 años en Alemania, Australia, Austria, Brasil, Dinamarca, España, Estados Unidos, Finlandia, Holanda, Inglaterra, Italia, México, Nueva Zelanda, Panamá, Perú, Suecia y Suiza (Jurist, 2018, p. 92).

Distintos autores, en publicaciones diversas, se han preguntado por su relación con el psicoanálisis y sus respuestas han sido también diversas (Allen, Fonagy, Bateman, 2008; Holmes, 2006; Kernberg, 2012; Kirsch, 2019; Leuzinger-Bohleber, 2008; Taubner, 2015; Vermote et al., 2012).

Parafraseando el título de un Journal que dedicó todo un número a este tema (Journal für Psychoanalyse, 2011), cabría decir que el debate sigue abierto y que es mucho todavía lo que queda por reflexionar al respecto.

Por mi parte, considero que una forma posible -entre otras- de reflexionar acerca de esta relación, consiste en circunscribir algunos temas teórico-clínicos específicos para interrogarse respecto a cómo son vistos desde un punto de vista y desde el otro y cuáles son las consecuencias de estas diferencias en el abordaje clínico concreto. A su vez, a partir de estos temas así delimitados, cabe también preguntarse si es posible una articulación o complementación teórico-clínica entre ambos enfoques, al menos en lo que tiene que ver con ese tema específico.

De este modo, tratando de llevar adelante una reflexión como la mencionada, deseo focalizar en un tema que se halla en el centro de los estudios de las últimas décadas, tanto de aquellos provenientes de la teoría del apego (Dornes, 2000; Fearon et al., 2014; Fonagy, 2001; Fonagy, Campbell, 2015; Fonagy, Target, 2007; Holmes, 2010), como de los surgidos del ámbito de la investigación del desarrollo temprano (Dornes, 1993, 1997, 2000, 2003, 2006; Gergely \& Watson, 1996; Stern 1985), como de los producidos en un campo psicoanalítico pluralista, que busca integrar aportes diversos del ámbito del psicoanálisis, con las propuestas de los investigadores del desarrollo, la teoría social y las neurociencias (Bohleber, 1992, 2010).

Me refiero a la temprana relación madre-hijo, y dentro de ella a aquel aspecto de la misma mencionado por Winnicott en su trabajo sobre "El rostro materno..." cuando dice: "¿Qué es lo que ve el bebé cuando mira el rostro de la madre? Yo sugiero que por lo general se ve a sí mismo. En otras palabras, la madre lo mira y lo que ella parece se relaciona con lo que ve en él" (Winnicott, D. W., 1967, p. 112). 
Tenemos así acotado el siguiente tema: la madre como espejo para el niño y los procesos implicados en el reflejo materno y sus desenlaces, tanto en el desarrollo normal como en el patológico.

En lo que sigue analizaré de qué manera han conceptualizado Fonagy y colaboradores este tema, posteriormente caracterizaré de qué modo ha sido hecho desde el psicoanálisis. Tras establecer las comparaciones entre uno y otro enfoque, trataré de ilustrar los alcances clínicos de estas diferencias con una viñeta, a partir de la cual podremos preguntarnos si es posible encontrar una complementariedad entre ambos puntos de vista.

\section{El enfoque de Fonagy y colaboradores}

Fonagy y colaboradores hacen una referencia explícita a esta pregunta de Winnicott en su libro del año 2002. Es importante subrayar que las consideraciones que llevan a cabo en este último tienen lugar en el contexto de su interés por dilucidar los orígenes y naturaleza de la capacidad del niño para identificar y atribuir estados mentales, tanto a sí mismo como a los demás (2002, p. 146). O sea, éste es el punto de vista específico que guía sus consideraciones al respecto.

Su planteo se desarrolla en contraposición al de Meltzoff y Gopnik, quienes postulan una serie de habilidades innatas en el niño, entre ellas la de tener un acceso introspectivo directo a sus estados afectivos (1993).

Fonagy y colaboradores, por su parte, postulan que dicho acceso no es directo, sino que debe mediatizarse a través de la relación con la madre o el cuidador.

En consonancia con dicho planteo, postulan también que el sistema perceptivo del niño tiene un sesgo inicial según el cual está dirigido a prestar atención a los estímulos externos, más que a los internos. Por esa razón, el conjunto de claves internas, viscerales y propioceptivas que se activan cuando el niño tiene un estado emocional, no son percibidas inicialmente por éste, o al menos no son agrupadas de modo tal que puedan ser identificadas como un estado emocional específico y diferenciado.

Para llegar a dicha agrupación e identificación es esencial -según este enfoque- que tenga lugar una presentación repetida de un reflejo externo de las expresiones emocionales que el niño manifiesta cada vez que en él se activa una emoción determinada.

Basándose en los trabajos de Gergely y Watson $(1996,1999)$ Fonagy y colaboradores (entre los que se encuentra Gergely) postulan la teoría del biofeedback social para explicar este logro por parte del niño (Fonagy et al., 2002). 
En la medida en que utilizan el procedimiento del biofeedback para explicar su teoría, será de utilidad realizar una breve caracterización de dicho procedimiento, que se lleva a cabo con la ayuda de un sencillo aparato.

El sujeto que lo utiliza se conecta al mismo a través de una serie de electrodos que miden su presión arterial, su frecuencia cardíaca, la conductividad eléctrica de la piel, la tensión de su musculatura y otros procesos que dependen del sistema nervioso autónomo y que no suelen ser percibidos y mucho menos son pasibles de control voluntario.

La información recogida por los electrodos es enviada a un monitor, en el cual el sujeto puede ver la figuración visual de dichos procesos (por ejemplo, de su frecuencia cardíaca), por lo que podríamos decir que la misma es externalizada y observada, o que en dicho monitor encuentra un reflejo de dicha frecuencia.

La experiencia repetida de la percepción de dicho reflejo facilita que el sujeto vaya percibiendo en sí mismo y tomando conciencia, progresivamente, de los procesos mencionados. Dicha conciencia de sí, entonces, es mediatizada por la aprehensión del reflejo de sus propios estados que le proporciona el monitor.

Con un entrenamiento mayor podrá incidir voluntariamente en la intensidad de éstos, con lo que logrará un control deliberado de los mismos y un sentimiento de agencia al respecto.

Pues bien, Fonagy y colaboradores postulan que los mecanismos psicológicos implicados en el reflejo por parte de la madre son similares a los que se encuentran presentes en el biofeedback mencionado.

Para mayor claridad podríamos sintetizar y resumir en 9 pasos los procesos que tienen lugar en este entrenamiento natural en un biofeedback social, que postulan Fonagy et al. (2002).

1) El niño tiene una emoción que no identifica con claridad, pero que expresa (diversos estudios han demostrado que hay una conexión innata entre determinadas emociones y expresiones específicas que les corresponden). Esta emoción puede ser, o no, una de las emociones básicas innatas (al menos, placer o disfrute, rabia, temor, tristeza, asco y sorpresa) que son expresadas -cada una de ellas- por los mismos patrones faciales de acción muscular en las distintas culturas (Fonagy et al., 2002, p. 155).

2) La madre (o el cuidador) mentaliza al niño, esto es, empatiza con sus estados afectivos, que reconoce y suele compartir por vía de identificación. De este modo, la capacidad de mentalizar de la madre le permitirá identificar adecuadamente el afecto experimentado por el niño, resonando con él (Stern, 1985). A partir de ello será capaz de llevar a cabo un reflejo congruente con el estado interno de su hijo. 
3) La madre refleja la expresión del niño, lo que es un hecho comprobado también en las diversas culturas (Fonagy et al., 2002).

4) Este reflejo tiene un carácter "marcado". Esto significa que el reflejo no es una copia exacta de la expresión del niño, sino que resulta artificial y exagerado en relación a la misma, tanto en el patrón de entonación vocal, como en los rasgos faciales de la expresión emocional, visualmente perceptibles.

A su vez, la contención emocional que lleva a cabo la madre implica que ésta agregue, de manera sutil, un afecto de sentido contrario cuando el afecto primario vivenciado por el niño es displacentero (por ejemplo, combinando el temor con la serenidad).

5) Debido al carácter marcado del reflejo, el niño inhibe la atribución de dicha expresión a la madre, con lo que se desacopla dicha expresión de su referente, en lo que Fonagy et al. denominan desacople referencial.

Otra razón para dicho desacople es que el niño tiene un cierto grado de control sobre la expresión reflejante de la madre cuando ésta se produce en respuesta a su expresión emocional, ya que concuerda en el tiempo con ella, mientras que tal cosa no ocurre en el caso de la genuina expresión emocional de la madre.

6) No obstante, la expresión de la madre -así desacoplada de ésta- necesita ser interpretada por el niño desde un punto de vista referencial, esto es, como expresión de la emoción de alguien. Debido al alto grado de concordancia entre sus expresiones emocionales y el reflejo materno, el niño se ve llevado a realizar un anclaje referencial, lo que implica que comprende dicho reflejo como referido a sus propios estados afectivos.

7) Hay dos expresiones maternas que el niño aprende a diferenciar: aquella que expresa un estado afectivo de la madre y aquella que refleja su propio (del niño) estado emocional.

Es posible conjeturar entonces que, a partir de esa diferenciación, llega a construir una representación separada para esta última (la expresión que lo refleja), que posteriormente será internalizada.

Debido a la concordancia temporal de dicha representación con las reacciones afectivas automáticas del niño, esta representación ha de retener su lazo asociativo con dichas reacciones primarias y ha de funcionar como una estructura representacional secundaria de las mismas. 
8) Dicha estructura representacional se activará -a partir de entonces- cada vez que se activen las claves internas correspondientes a una emoción determinada.

Por lo tanto, el surgimiento de un estado emocional derivará en la activación automática de esta representación secundaria de la emoción, en la conciencia del niño.

9) La mencionada internalización de esta representación por parte de éste, le posibilitará aprehender y diferenciar su propio estado emocional, aún en ausencia de la madre.

El proceso caracterizado hasta este punto tendrá las siguientes consecuencias:

a) el niño llegará a detectar y a agrupar el conjunto de estados internos que corresponden a cada una de las diversas categorías emocionales diferentes (temor, rabia, alegría, etc.).

b) establecerá representaciones secundarias, asociadas con las reacciones primarias, lo que le proveerá de la posibilidad de atribuir los estados emocionales al self y de la capacidad de aprehenderlos con mayor claridad.

c) estas representaciones se encontrarán en la base del sistema representacional para los estados mentales, que se construirá con posterioridad, a lo largo del desarrollo.

d) dichas representaciones le serán de utilidad para llevar a cabo la regulación emocional, para la cual requiere inicialmente de diversas actitudes por parte de la madre (mecerlo, tocarlo, hablarle, reflejarlo, etc.).

En lo que hace al reflejo del afecto que venimos considerando, cabe reiterar que una razón para que el mismo actúe como regulador emocional es su carácter de marcado, en tanto integra la expresión de un afecto contrario al afecto displacentero que se halla activado en el niño (combinando, por ejemplo, la expresión de temor con la de serenidad) (Taubner, 2015).

Otra razón la encuentran Fonagy y colaboradores en la eficacia causal que el niño ha de atribuir a su expresión emocional (por ej. el llanto), en la medida en que la misma determina la asistencia de la madre, incluido su reflejo del afecto. Este control sobre el comportamiento de la madre ha de inducir un estado positivo en el infante, el cual, por vía de inhibición recíproca, ha de hacer decrecer su afecto displacentero.

Al experimentar la eficacia causal de su expresión emocional en el sentido mencionado, el infante ha de registrar también la modificación positiva de su estado afectivo, consecuencia de la asistencia de la madre. De este modo, en la medida que esta situación 
se repite una y otra vez, se van echando las bases para que el infante experimente paulatinamente un sentimiento de sí como agente autorregulador.

Un segundo nivel en esta regulación tiene lugar cuando se internalizan las representaciones del rostro materno -tal como fue señalado más arriba-. De este modo, la capacidad de regulación que antes dependía de la presencia efectiva de la madre, se independiza poco a poco de la misma, de modo tal que el niño puede paulatinamente hacer uso de la representación mencionada, a los efectos de la autorregulación emocional.

Si tomamos en cuenta ahora el modo en que desde este punto de vista se hipotetiza acerca de la construcción del self psicológico, diríamos que son las representaciones de la propia experiencia emocional las que se encuentran en la base de dicha construcción.

En palabras de Bateman y Fonagy, podríamos decir que: "El cuidador que es capaz de dar forma y significado a los estados afectivos e intencionales del niño pequeño a través del reflejo facial y vocal y de interacciones lúdicas, provee al niño con representaciones que han de formar el núcleo de su sentido del self en desarrollo.

Para su desarrollo normal, el niño necesita experimentar una mente que tenga a su mente en mente y que sea capaz de reflejar sus sentimientos e intenciones adecuadamente y de un modo no abrumador" (Bateman, Fonagy, 2004, p. 68).

Vale decir que el infans ve que el cuidador se lo representa como un ser intencional (poseedor de estados mentales intencionales) y es, entonces, esta representación la que es posteriormente internalizada para formar el núcleo del self psicológico. "Pienso, luego soy" no parece, por tanto, ser un modelo psicodinámico adecuado del nacimiento del self. "Ella piensa que yo pienso, por lo tanto existo como un ser pensante" se halla probablemente más cerca de la verdad de esta experiencia intersubjetiva, que se encuentra en la base de la formación del self (Fonagy et al., 2002).

Si quisiéramos sintetizar lo expresado hasta aquí, podríamos decir que en base a las consideraciones consignadas advertimos que el punto de vista de la teoría de la mentalización busca comprender las siguientes cuestiones:

a) Cómo llega el niño a tomar conciencia de sus propios estados emocionales.

b) Cómo logra regular dichos estados emocionales.

c) Cómo se inicia la construcción de un sistema representacional para los estados mentales.

d) Cómo se construye el self psicológico. 
La situación de la madre reflejando al niño y a sus estados mentales es visualizada desde estos interrogantes, que son los privilegiados en este punto de vista.

Si dirigimos nuestra atención ahora a algunos posibles desenlaces patológicos de este proceso, diremos que cuando el reflejo no es marcado, la representación secundaria del estado emocional primario del niño no se establece, por lo que tienen lugar posteriormente deficiencias en la percepción de sí y en la simbolización y regulación de los afectos. Asimismo, al tomar a la expresión materna como manifestación de estados afectivos negativos de la madre, en respuesta a sus propios estados negativos, estos escalarán y conducirán a una situación traumática más que a una contención.

Cuando lo que falla es la congruencia con el estado emocional del niño, la consecuencia será una representación de sí distorsionada. Este desenlace vincula este modelo con el concepto de falso self de Winnicott (Fonagy et al., 2002, pp. 193-196).

De igual forma, cuando el niño no puede encontrarse a sí mismo en la mente de los cuidadores, esto es, cuando éstos fallan en proveerle un reflejo adecuado de símismo como un ser intencional, cuando además abusan de él, lo maltratan o abandonan, se produce un fenómeno consistente en que lo que el niño internaliza como parte de su representación de sí, no es su propio reflejo, sino la imagen del cuidador, la cual pasa, entonces, a formar parte del propio self. De este modo, ideas y sentimientos que no parecen pertenecer al self, son experimentados como parte del mismo.

Esta internalización de los cuidadores malevolentes o abusadores como un "self ajeno" se expresa como la separación episódica de un sentido de propiedad o identidad con las propias acciones o experiencias -algo que es en realidad hecho por "mi" (Me) pero que no siento como si "yo" (I) lo hiciera, lo cual es una experiencia habitual en los pacientes borderline. Esta discontinuidad en la experiencia del self, esta fragmentación en su estructura busca ser remediada a través de la identificación proyectiva. Merced a este proceso, esta parte discordante del self es ubicada en un otro, con lo cual el self puede recuperar un sentimiento de coherencia e integración.

\section{El punto de vista psicoanalítico:}

Vale la pena aclarar que debido al pluralismo existente hoy en día en psicoanálisis, no es posible hablar de "el" punto de vista psicoanalítico, ya que no nos encontramos con "un" psicoanálisis, sino con desarrollos distintos dentro de este amplio campo (Bernardi, 1994i Bohleber, 2019; Jimenez, 2006; Wallerstein, 1988, 1990). 
Por esa razón, llevaré a cabo una opción teórica articulando las propuestas de Donald Winnicott, Heinz Kohut, René Roussillon y Johann Jung, las cuales poseen múltiples elementos comunes, así como otras tantas diferencias.

El punto de vista que tomo en consideración a partir del cual los autores mencionados han reflexionado acerca de la situación que nos ocupa, es el del narcisismo, concepto complejo que incluye distintos aspectos.

En el planteo de Winnicott, el que el niño mire el rostro de la madre y vea que ésta lo ve, tiene que ver con su sentimiento de existencia: "Cuando miro se me ve, y por lo tanto existo" (1967, p. 151). Nos encontramos, por tanto, en el terreno de la constitución de sí, que posteriormente relacionará con el verdadero self, el sentimiento de continuidad de existir, etc. Se trata de que el niño sea visto y de que el rostro materno le devuelva al niño su persona (1967, p. 155).

Podríamos decir entonces, de un modo harto esquemático, que la hipótesis central de Winnicott en este punto consiste en que el objeto primario (entendido como otro sujeto) es anhelado por el sujeto como un espejo afectivo y representativo de sí mismo. De este modo, la identidad se constituirá -desde este punto de vista- en un movimiento de recuperación del reflejo proveniente del otro.

La identidad es considerada entonces, como un precipitado de las identificaciones narcisistas primarias, identificaciones con aquello que el objeto primordial ha reflejado del (y al) sujeto (Roussillon, 2001, 2011).

Cuando esto no sucede, cuando el niño no se encuentra reflejado en el rostro del objeto primordial, las consecuencias puede ir desde el no sentirse real hasta experiencias de despersonalización y desintegración (Kohut, 1977).

Sin embargo, con anterioridad (y con posterioridad) a esta situación -del reflejo maternohay otras situaciones que poseen la mayor importancia.

Si quisiéramos ahora establecer una serie de momentos, tal como hicimos con la postura de Fonagy y colaboradores, podríamos esquematizarlos del siguiente modo:

1) En un primer momento, que denominamos de narcisismo primario (Kohut, 1966; Roussillon, 2001, 2011) el niño no vive a la madre y sus cuidados como un "tú", sino que lo hace en el contexto de una vivencia del mundo en la que la diferenciación entre el yo y el tú no ha tenido lugar aún (Kohut, 1966). La madre se relaciona con el hijo a través del holding y el handling (Winnicott), del cuidado físico, las caricias que prodiga, el calor de su cuerpo, el arrullo de su voz, etc. 
En el niño la experiencia es esencialmente física y de emociones primarias poco diferenciadas de las sensaciones corporales.

En el adulto, una experiencia en algún sentido comparable podría tener lugar en aquellas situaciones en que los límites entre el yo y el mundo exterior parecen desvanecerse, como en el estado de enamoramiento y en el sentimiento oceánico (Freud, 1930, pp. 65-69) y posiblemente también en la transferencia fusional (Kohut, 1971) que puede acontecer dentro o fuera del análisis.

2) Paulatinamente tiene lugar una gradual separación entre la madre y el hijo, lo que da lugar a eventuales angustias de separación y desintegración (Green, 1982), que pueden ser mantenidas a raya por la presencia de la madre (Winnicott, 1962). Es el tiempo del autoerotismo en Freud (1914) y de los núcleos del Self en Kohut (1977).

Cabe aclarar que la madre se dirige al hijo desde el principio como a una totalidad: al satisfacer el hambre de éste no se relaciona con una boca y un estómago, sino con su bebé (que tiene un nombre) que tiene hambre (Kohut, 1977, p. 34). Con ello anticipa y promueve la unificación del self.

3) En un momento posterior, en el que ha avanzado la diferenciación entre el niño y su madre, y en el que los núcleos del self se encuentran en proceso de integración en una unidad, tiene lugar la función de la madre como espejo para el niño, un espejo que no sólo refleja, sino que lo hace amorosamente, invistiendo al niño con su amor y su libido idealizadora. Kohut habla del "brillo afectuoso en los ojos de la madre" (Elson, 1987, p. 59).

4) El niño, por su parte, se encuentra en esa imagen de sí que recibe de su madre, lo que le permite la unificación de su self y la construcción de un Yo-ldeal (o Self grandioso) (Kohut, 1971, p. 40; Winnicott, 1962).

Este Self grandioso posee tendencias exhibicionistas que son confirmadas por la empatía materna, con lo que se consolida el Self nuclear, base de la autoestima posterior.

La imagen de sí, que es inicialmente externa (lo que se refleja en los ojos de la madre) será posteriormente internalizada y consolidará la unificación del self así como su consistencia. Este self, a su vez, será investido con libido (narcisista) por el propio niño (además de por la madre), lo que es esencial para el mantenimiento de su consistencia y cohesión, como así también para su autovaloración.

5) Paulatinamente se irá modificando este sentimiento de grandiosidad al contacto con la experiencia y con las propias limitaciones. "Bajo condiciones óptimas de desarrollo, 
el exhibicionismo y la omnipotencia del Self grandioso arcaico son progresivamente dominados, hasta que por último toda la estructura se integra en la personalidad adulta y proporciona energía instintiva para nuestras ambiciones y propósitos egosintónicos, para disfrutar de nuestras actividades y para importantes aspectos de nuestra autoestima" (Kohut, 1971, p. 42).

6) Se proyecta entonces la grandiosidad perdida en la imago parental idealizada, (según Kohut, estas dos líneas se san simultáneamente, 1971, p. 42).

7) En un momento posterior se morigera la idealización parental y se produce paulatinamente una internalización de la misma, de sus funciones, las que son idealizadas por libido narcisista. Se forma entonces el Ideal del Yo, cuyos valores son supremos en tanto se halla investido por libido narcisista y, por tanto, idealizado.

Esto supone la construcción de una estructura que posee una serie de funciones.

El resultado de este proceso -cuando tiene lugar de un modo óptimo- es la constitución de un self cohesivo, integrado, vital y creativo, así como de ideales y ambiciones acordes a los talentos y capacidades del Yo y a la situación vital e interpersonal en que éste se encuentra (Kohut, 1971, 1977).

La diferenciación de estos distintos momentos resulta de utilidad a los efectos de puntualizar que resultan diferentes los desenlaces patológicos según tenga lugar una perturbación en la relación madre-hijo, por ejemplo, en el punto 1 (Smadja, 2001; Winnicott, 1962), que en el punto 3 (Kohut, 1977). Ésta es una de las diferencias entre el punto de vista de Fonagy y el psicoanalítico, ya que aquél no conceptualiza perturbaciones anteriores a la de las fallas en el reflejo parental, mientras que el psicoanálisis sí lo hace.

Menciono ahora algunos de los desenlaces que tienen lugar cuando ha habido una perturbación en el punto 3, a los efectos de compararlos con los que postula Fonagy como corolario de una falla en la función especular de la madre.

Entre ellos encontramos la debilidad y vulnerabilidad del self, junto a una tendencia a caer temporariamente en estados de fragmentación, perturbaciones profundas en el sentimiento de sí y en el sentimiento de estima de sí (autovaloración) así como en su regulación, lo que vuelve al sujeto dependiente del auxilio ajeno para restañar dicho sentimiento. De igual forma observamos experiencias de vacío, de muerte anímica, de falta de energía y vitalidad, de no sentir el self como real, de insuficiencia y pequeñez, así como de una grandiosidad difícil de modular debido a fallas en las estructuras moduladoras. En este último caso suele apelarse a defensas frágiles, del tipo "todo o nada", siendo reprimida la grandiosidad, con lo cual el resto de la personalidad se empobrece, en la medida en que 
no puede apropiarse de la energía libidinal de la misma, o irrumpe en el comportamiento de diversas formas, perturbando las relaciones interpersonales (Kohut, 1971, 1977).

Otras defensas apelan a la activación de las pulsiones, a los efectos de lograr un sentimiento de sí e intentar neutralizar el vacío. De este modo encontramos, por ejemplo, una masturbación compulsiva acompañada eventualmente de fantasías sadomasoquistas, o comportamientos perversos y/o promiscuos, así como adicción a las drogas o trastornos en la alimentación.

Asimismo encontramos perturbaciones en el terreno de las ambiciones y los ideales de diversa índole. Una de ella, muy habitual, consiste en que no se logra una internalización adecuada de los Ideales, por lo que la persona depende de que un "líder" le indique el camino a seguir y le dé ánimos para permanecer en él (Kohut, 1971).

Cabe agregar que los desarrollos en torno al narcisismo en el campo psicoanalítico han dado lugar a una profusa literatura, que incluye temas como: la integridad del self, las formas normales y patológicas de regulación de la autoestima, los narcisistas de piel fina y de piel gruesa, la patología del narcisismo en su relación con la transferencia, la rabia narcisista, las defensas contra la debilidad del self y su tendencia a la fragmentación, el narcisismo de vida y de muerte, el narcisismo maligno, etc.

Todo este complejo desarrollo teórico encuentra su origen en el trabajo de Freud de 1914 y supone un punto de vista específico sobre el self, su integridad y su autovaloración, el tema de las identificaciones (primarias y secundarias), así como sobre las defensas del narcisismo, las estructuras compensatorias, etc. A su vez, permite comprender y enfocar terapéuticamente un sinnúmero de perturbaciones que tienen lugar en este territorio.

Todos estos temas están ausentes de las consideraciones de la teoría de la mentalización sobre las fallas en el reflejo materno. Correlativamente, las conclusiones obtenidas desde el punto de vista de esta teoría, señaladas más arriba, no se encuentran presentes -de ese modo- en el marco teórico del psicoanálisis, si bien en la obra de Bion encontramos algunas consideraciones análogas (Bion, 1962).

Las diferencias en las conclusiones a las que se llega en un caso y en otro, parecen obedecer a los puntos de vista diferentes y específicos de cada enfoque, a aquellos aspectos de la situación que cada enfoque resalta y busca comprender, relacionados a su vez con conjuntos de conceptos también propios de cada uno de ellos.

De todos modos, estas diferencias no suponen contradicción o mutua exclusión entre ambas perspectivas, sino que podemos encontrar en ellas una complementariedad que puede resultar de utilidad en la práctica clínica. 
Para ilustrar de algún modo dicha complementariedad, consigno en lo que sigue una viñeta clínica y llevo a cabo algunos comentarios sobre la misma.

\section{Ejemplo clínico:}

En aras de la protección de la intimidad de las personas que me han requerido para un trabajo psicoanalítico, he de utilizar como ilustración clínica una situación entre una adolescente y sus padres que es de público conocimiento, ya que ha sido descripta en una novela autobiográfica (Latini, 2006).

Dicha situación, narrada en las primeras páginas de la novela, se encuentra precedida por una serie de consideraciones que revisten el mayor interés psicológico (sobre los estados emocionales de la autora, su falta de amigas, la importancia de la opinión de los otros, su gordura, las humillaciones padecidas, etc. En un pasaje dice: "En realidad, yo (...) me sentía mal, entonces todo lo que hacía era comer. Mis compañeras del colegio jugaban a la soga y yo comía, mis compañeros jugaban al fútbol y yo comía, ellos eran perfectos alumnos y yo comía").

Tras estas consideraciones, dice: "Y siguiendo con mis traumas, recuerdo a mis viejos" Relata a continuación una escena en la mesa en que el padre le dice que no coma mayonesa porque engorda, tras lo cual expresa:

"Mis padres me decían qué tenía que comer y qué no. Se empezaron a preocupar por mi aspecto físico, pero jamás se preocuparon porque yo no tenía amigas, porque leía demasiado, porque no recibía llamadas telefónicas ni quería festejar mi cumpleaños. Esas cosas parecían no interesarles y se escudaban en la siguiente frase: es una nena especial"

Relata a continuación que la madre la envió a tomar clases de piano, en las que resultó sobresaliente. "No solamente era una excelente alumna de piano, sino que era el orgullo de mi familia (...) Siempre que venía algún invitado me pedían que tocara una invención de Bach o alguna sonata, lo cual no me gustaba ni un poco, pero lo hacía. Yo pensaba que me querían porque tocaba piano"

(...) "Mis habilidades eran muchísimas: danza, tenis, piano, natación, inglés"

"Siempre tuve la creencia, equivocada o no, de que mi mamá quería que yo fuera una diez"

Comenta a continuación que una pareja amiga de los padres tenía una hija diez, llamada Rocío. "El panorama se me complicó un poco cuando empecé a escuchar a mamá diciendo a cada rato que algún hijo perfecto de su amiga había recibido algún estúpido premio. Me empezó a molestar la repetición en serie de comentarios edulcorados. Como ella estudiaba 
inglés, mi mamá me mandó a estudiar inglés. Como ella bailaba danzas contemporáneas, yo empecé a hacerlo. $Y$ así seguía como un detective frustrado tras las huellas de Rocío. $\mathrm{O}$ mejor: cumplía los caprichos de mi madre.

Gracias a Rocío mis habilidades eran innumerables. Era una vulgar fotocopia de mi compañera del colegio".

Entre otras consideraciones que podrían hacerse sobre estos fragmentos, considero que resultará de interés -retomando las ideas expresadas con anterioridad- llevar a cabo dos clases de reflexiones teórico-clínicas: unas basadas en el punto de vista psicoanalítico y otras en la teoría de la mentalización.

Desde el punto de vista psicoanalítico podríamos decir que Cielo parece ser para su madre una extensión narcisista (al menos desde la perspectiva de Cielo, que es la que seguiré en todas mis reflexiones) y una "posesión narcisista". Esto último significa que el valor que la madre atribuye a su hija a raíz de sus logros, recae sobre el yo de la progenitora, realzándolo en su valor, en su sentimiento de estima de sí (Bleichmar, 1983).

En tanto la vive como posesión narcisista decide sobre ella, enviándola a aprender inglés, danza contemporánea, etc., sin considerar los deseos de su hija, sus gustos e inclinaciones.

Podemos conjeturar, entonces, que la madre de Cielo intenta compensar su propio narcisismo, posiblemente deficitario, a través de tener una hija "diez" (como la de su amiga). Por esa razón, viviendo a Cielo como una extensión de sí misma, la envía a adquirir distintas habilidades que la asemejarían a Rocío (hija perfecta y premiada). Con ello dificulta que Cielo lleve a cabo los procesos de separación e individuación como parte de la construcción de su identidad, para lo cual necesitaría -entre otros factores- una madre que la refleje y la invista como un ser diferente, con un valor en sí mismo, destinado a tener una autonomía y una vida propias.

Otras variables que -desde este punto de vista- serían importantes para contextualizar esta relación madre-hija son: la historia de la madre de Cielo con su propia madre, la posible falta de realizaciones personales y la relación que mantiene con su esposo.

Asimismo, parece de interés la relación de la madre de Cielo con la madre de Rocío, con quien parece mantener un vínculo de rivalidad envidiosa. Si así fuera, en las actitudes con su hija (mandarla a que estudie lo que estudia Rocío) parecería estar intentando neutralizar su envidia a través de equiparar a su hija con la hija de su amiga.

En lo que hace al rol del padre en este contexto, podríamos conjeturar que se encuentra en déficit una función paterna que podría promover un corte entre madre e hija. 
Si ponemos ahora el foco en lo que Cielo dice de sí misma, vemos que se conjugan en ella el sentimiento de no ser amada por ella misma sino por la gratificación narcisista que es capaz de proporcionar a su madre, con un profundo malestar que no alcanza a ser simbolizado y se tramita a través de una ingesta desenfrenada.

A la vez, no es difícil observar su sujeción al deseo materno y su ubicación como aquella que realza el narcisismo familiar ("era el orgullo de mi familia").

Convergen entonces en ella la sobreadaptación, la retracción (falta de amigas), los déficits en la capacidad de simbolizar y el recurso a la ingesta en un intento de neutralizar su malestar ("...no era que no tenía amigas porque era gorda, sino que era gorda porque no tenía amigas").

Si consideramos ahora su identidad, podríamos decir que parece haber construido un falso self (Winnicott, 1960), que encarna los deseos maternos de que ella fuera una hija "diez", como Rocío. Cielo, entonces, lleva a cabo una serie de actividades y se mimetiza (fotocopia) con Rocío, adquiriendo así una identidad prestada para satisfacer el narcisismo materno y sentir un sucedáneo del amor de la madre, en el orgullo que ésta siente por los desempeños de su hija.

Siguiendo, entonces, con las consideraciones que hemos expuesto al hablar del rostro materno considerado desde el punto de vista psicoanalítico, he puesto básicamente al acento en las conjeturas que podemos construir si tomamos como eje el tema del narcisismo.

Si enfocamos ahora esta relación desde el punto de vista de la teoría de la mentalización, podríamos decir que la madre no ha logrado una adecuada diferenciación sujeto-objeto, al menos en la relación con su hija. También diríamos que tanto ella como su esposo parecen tener marcados déficits en su capacidad de mentalizar, lo que implica que no son capaces de formarse un modelo de los estados mentales que determinan el comportamiento de Cielo. Parecería que sólo pueden ver los aspectos físicos y externos de su hija, sin ser capaces de aprehender su mundo interno o de interrogarse por el significado vivencial que podían tener distintas características, vinculares y personales de la misma ("Se empezaron a preocupar por mi aspecto físico, pero jamás se preocuparon porque yo no tenía amigas, porque leía demasiado, porque no recibía llamadas telefónicas ni quería festejar mi cumpleaños").

Cuando intentan explicarse el por qué de este proceder de Cielo no pueden emplear una explicación basada en la aprehensión de los estados mentales (motivaciones, sentimientos, conflictos, etc.) de su hija, sino que apelan a un rótulo que no incluye dicha referencia: "es 
una nena especial". De este modo, la subjetividad de Cielo permanece para ellos en la más completa opacidad, ya que no dirigen su atención sobre el mundo interno de la misma, sino que lo hacen, como fue dicho, sobre lo externo de su aspecto físico. Su falla en el mentalizar consistiría en poner el acento exclusivamente en el polo "procesos centrados en lo externo", dentro de la polaridad procesos centrados en lo interno-procesos centrados en lo externo, en lugar de poder moverse flexiblemente entre uno y otro polo (Taubner, 2015).

Esta falla se relaciona con el punto 2) mencionado al hablar del enfoque de la teoría de la mentalización, que, si bien tiene lugar en lo actual, podríamos conjeturar que ha tenido lugar también en los primeros tiempos de la vida de Cielo, dadas las dificultades que parece haber tenido para construir representaciones secundarias para simbolizar sus afectos.

Si nos remitimos a los conceptos expresados más arriba, diriamos que esta falla en la capacidad mentalizadora de los padres tiene habitualmente profundas implicancias en el hijo. En la medida en que éste no puede encontrar en la mente de los padres una imagen de sí como alguien con deseos, intenciones, afectos, impulsos...en suma, estados mentales, carece de una pieza clave para la construcción de su self psicológico, que suele basarse en la internalización de esa imagen que los padres han construido de él (tal como fue señalado más arriba). De ahí que -en el caso de Cielo- ésta no pueda alcanzar una "identidad" y se vea como una "fotocopia" de su compañera de colegio.

Por otra parte, la mencionada falla de los padres, dificulta que Cielo construya las representaciones secundarias mencionadas con anterioridad y desarrolle su propia capacidad de mentalizar, por lo que le resulta en extremo difícil identificar y regular su malestar simbolizándolo mediante las representaciones secundarias para simbolizar los afectos (Fonagy et al., 2002), de ahí que intente neutralizarlo a través de la ingesta.

Vemos, por tanto, que ambos enfoques se solapan en algún punto (déficit en la capacidad de simbolizar), se asemejan en otro punto (fallas en la construcción de la identidad, entendida de modos distintos en un enfoque y en el otro) y se diferencian en otros, pero no entran en contradicción y más bien podríamos pensar que entre ambos es posible encontrar interesantes complementariedades.

Por lo demás, la articulación de ambos puntos de vista focalizando sobre Cielo y sus padres, permite una visión tridimensional, por así decir, a partir de la cual se puede ir y venir desde una perspectiva a la otra, a la vez que es posible intentar aprehender cómo se imbrican.

Una posible imbricación entre dichos puntos de vista -en torno al problema que nos ocupacabría encontrar postulando que el adecuado mentalizar materno se entrelaza siempre con una investidura amorosa e idealizadora del hijo (base del narcisismo logrado de éste), 
vivido como diferente de sí misma, de modo tal que hace las veces de una precondición y un complemento de la aprehensión del hijo como ser intencional. Por el contrario, una investidura deficiente del hijo, o el que éste sea vivido como una extensión de sí misma (con la perturbación narcisista que conlleva), dificultaría la puesta en juego del mentalizar y, por tanto, de la aprehensión de los estados mentales efectivos del niño.

Cabe conjeturar entonces que las fallas -cuando las hay- han de producirse en ambos ejes, el del narcisismo y el de la mentalización, por lo cual ha de ser en aquellos pacientes que tienen fallas en la constitución de su narcisismo, de sus identificaciones primarias, en quienes encontraremos también fallas en el mentalizar.

Por último, podríamos preguntarnos cómo trabajaríamos con estos padres y con su hija, si hubiesen consultado por Cielo, ya que el trabajo con los padres es esencial en la clínica con este tipo de adolescentes.

Si enfocáramos la situación desde el punto de vista de la mentalización, consideraríamos importante ayudar a la madre a diferenciarse de su hija, y a ambos padres a que comiencen a conectarse con sus propios estados mentales, para poder hacerlo posteriormente con los de Cielo (se pondría el énfasis en la capacidad de mentalizar de los padres). Mediante una serie de técnicas específicas se buscaría ayudarlos a que puedan poner el foco no tanto en el aspecto físico de su hija, sino en su mundo interno, en sus emociones y en aquellos vínculos en los que éstas surgen (con ellos mismos, con sus compañeros de colegio, etc.). Esta tarea implicaría un movimiento de descentramiento, según el cual se buscaría que pudieran ver a su hija en sí misma, desde el punto de vista de ésta, a los efectos de poder empatizar con sus propias preocupaciones.

En el trabajo con Cielo se pondría el acento en favorecer y estimular sus capacidades para simbolizar e identificar los estados emocionales que la llevan a la ingesta desenfrenada, así como también en la dependencia en que se encuentra su self respecto de las expectativas de su madre para con ella.

$\mathrm{Si}$, de un modo complementario, enfocásemos la situación desde el punto de vista psicoanalítico, trabajaríamos sobre el significado que tienen para los padres los logros de su hija, de cómo alcanzan -a través de ella- un "orgullo familiar" del que posiblemente carecían. Asimismo, sería de utilidad tener en cuenta la relación entre los mismos y la satisfacción que alcanzan (o no) en dicha relación. De igual forma, habría que indagar sobre el lugar del padre en esa familia y en el deseo de la madre, relacionados con la dificultad que parece tener para cumplir su función. 
También sería de importancia explorar la relación que la madre mantiene con su amiga y los déficits narcisistas que la aquejan. En suma, pondríamos en foco todos aquellos elementos que hemos resaltado al considerar la situación desde el punto de vista psicoanalítico.

En lo que tiene que ver con Cielo el trabajo buscaría promover la toma de conciencia de su alienación en el deseo materno y su necesidad de conseguir por esta vía el sustituto de un amor inalcanzable de otro modo. Asimismo, se intentaría promover su subjetivación y su conquista de una identidad personal.

Las intervenciones que tendríamos en uno y en otro caso serían sin duda diferentes, en tanto lo son los objetivos que se plantean según se miren las cosas con la lente psicoanalítica o con la de la teoría de la mentalización.

De este modo, sería de interés trabajar tanto sobre las variables mencionadas desde el punto de vista de la mentalización como así también sobre aquellas puestas de manifiesto desde el punto de vista psicoanalítico.

Por último, cabe consignar que conocemos el grado en que la teoría hace de lente o filtro, que resalta unos aspectos y deja de lado otros, o que decide sobre el significado de un hecho clínico determinado. Y dado que ninguna teoría posee una visión omnisciente sobre las vicisitudes, características y problemáticas de los pacientes, resulta de utilidad la articulación de las mismas para enriquecer el espectro de intervenciones posibles. Los desarrollos vertidos a lo largo de este trabajo parecen indicar que tal cosa se torna posible también entre la teoría de la mentalización y el psicoanálisis, al menos en lo que tiene que ver con el tema específico considerado.

\section{REFERENCIAS}

Bateman, A., Fonagy, P. (2004). Psychotherapy for Borderline Personality Disorder. Mentalizationbased Treatment. Oxford: University Press

Bernardi, R. (1994). Sobre el pluralismo en psicoanálisis. Psicoanálisis APdeBA - Vol. XVI - No 3 433-455.

Bion, W. R. (1962). Learning from experience. London: Heineman; New York: Basic Books.

Bleichmar, H. (1983). El narcisismo. Estudio sobre la enunciación y la gramática inconsciente. Buenos Aires: Ediciones Nueva Visión.

Bohleber, W. (1992). Identität und Selbst. Die Bedeutung der neueren Entwicklungsforschung für die psychoanalytische Theorie des Selbst. Psyche, 1992, 46 (4), 336-365. 
Bohleber, W. (2010). Was Psychoanalyse heute leistet. Klett-Cotta 2012

Bohleber, W. (2019). Von der Orthodoxie zur Pluralität. Kontroversen über Schlüsselbegriffe der Psychoanalyse. Vandenhoeck \& Ruprecht.

Dornes, M. (1993). Der kompetente Säugling. Die präverbale Entwicklung des Menschen. Frankfurt am Main: Fischer Taschenbucher Verlag 7 Aufl. 1996

Dornes, M. (1997). Die frühe Kindheit. Entwicklungspsychologie der ersten Lebensjahre. Frankfurt am Main: Fischer Taschenbucher Verlag. 10 Auflage, 2013

Dornes, M. (2000). Die emotionale Welt des Kindes. Frankfurt am Main: Fischer Taschenbucher Verlag. 6 Auflage, 2014.

Dornes, M. (2003). Menschenbilder in Psycoanalyse und Säuglinsforschung. Konflikt oder Dialog? En Zwiebel, R., Leuzinger-Bohleber, M. ( $\mathrm{Hg})$ Träume, Spielräume II, Kreativität und Persönlichkeitsentwicklung. Göttingen : Vandenhoeck \& Ruprecht, 2003.

Dornes, M. (2006). Die Seele des Kindes. Entstehung und Entwicklung. Frankfurt am Main: Fischer Taschenbucher Verlag. 4 Auflage, 2013.

Elson, M. (comp) (1987). Los seminarios de Heinz Kohut. Sobre psicología del sí-mismo y psicoterapia con adultos jóvenes. Buenos Aires: Paidós, 1990.

Fearon, P., Shmueli-Goetz, Y., Viding, E., Fonagy, P. \& Plomin, R. (2014). Genetic and environmental influences on adolescent attachment. Journal of Child Psychology and Psychiatry

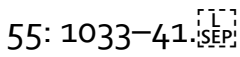

Fonagy, P. (2001). Attachment Theory and Psychoanalysis. New York: Other Press.

Fonagy, P., Campbell, C (2015). Bad blood revisited: Attachment and Psychoanalysis. British Journal of Psychotherapy 31, 2, 229-250.

Fonagy, P., Target, M. (2007). The Rooting of the Mind in the Body: New Links Between Attachment Theory and Psychoanalytic Thought J. Am. Psychoanal. Assoc. 2007; 55; 411

Fonagy, P., Gergely, G., Jurist, E., Target, M. (2002). Affect Regulation, Mentalization and the Developmento of the Self. New York: Other Press.

Gergely, G. \& Watson J. (1996). The social biofeedback theory of parental affect-mirroring: the development of emotional self-awareness and self- control in infancy International Journal of Psychoanalysis 77, 1181-1212

Gergely, G. \& Watson, J. (1999). Early social-emotional development: Contingency perception and the social biofeedback model. In: P. Rochat (Ed.) Early Social Cognition: Understanding Others in the First Months of Life (pp. 101-137) Hillsdale, NJ: Lawrence Erlbaum.

Green, A. (1982). Prólogo. El narcisismo y el psicoanálisis: ayer y hoy, en (1983) Narcisismo de vida, narcisismo de muerte. Amorrortu Editores, 1990.

Holmes, J. Holmes, J. (2006). Mentalizing from a Psychoanalytic Perspective: What's New? En Allen, J. \& Fonagy, P. (2006). Handbook of Mentalization-Based Treatment. UK: John Wiley

Holmes, J. (2010). Exploring in Security. Towards an attachment-Informed Psychoanalytic Psychotherapy. Routledge: East Sussex. 
Jimenez, J.P. (2006). After pluralism: Towards a new, integrated psychoanalytic paradigm. Int J. Psychoanal. 87: 1487-507.

Kernberg, O. (2012). The Inseparable Nature of Love and Agression. Clinical and Theoretical Perspectives. American Psychiatric Publishing. Washington DC.

Kohut, H. (1966). Formen und Umformungen des Narizissmus, en Die Zukunft der Psychoanalyse. Frankfurt am Main : Suhrkamp, 1975.

Kohut, H. (1971). Análisis del self. El tratamiento psicoanalítico de los trastornos narcisistas de la personalidad. Buenos Aires: Amorrortu editores, 1977.

Kohut, H. (1977). La restauración del sí mismo. Buenos Aires: Paidós, 1980

Kohut, H. (2002). Los dos análisis del Sr Z. Barcelona: Editorial Herder S.A., 2002.

Latini, C. (2006). Abzurdah. La perturbadora historia de una adolescente. Buenos Aires: Grupo editorial Planeta, 3ra edición, 2011.

Leuzinger-Bohleber, M. (2008). Biographical truths and their clinical consequences: Understanding 'embodied memories' in a third psychoanalysis with a traumatized patient recovered from severe poliomielitis. International Journal of Psychoanalysis, (2008) 89:11651187.

Leuzinger-Bohleber, M. (2009). Frühe Kindheit als Schicksal? Trauma, Embodiment, Soziale Desintegration, Psychoanalytische Perspecktiven.Stuttgart : Kohlhammer

Meins, E. (1997). Security of Attachment and the Social Development of Cognition. East Sussex, UK: Psychology Press.

Meltzoff A.N., Gopnik, A. (1993). The role of imitation in understanding persons and developing a theory of mind. En S. Baron Cohen, H. Tager-Flusberg y D. Cohen (Eds.) Understanding Other Minds: Perspectives from Autism (pp. 335-366). New York: Oxford University Press

Roussillon, R. (2001). Le plaisir et la répétition. Théorie deu processus psychique. Paris: Dunod, 2001.

Roussillon, R. (2011). Déconstruction du narcissisme primaire L'Anée psychanalytique internationale, 2011/1 Volume 2011, 177-193

Smadja, C. (2001). La vida operatoria. Estudios psicoanalíticos. Madrid: Biblioteca Nueva, 2005.

Stern, D. N. (1985). El mundo interpersonal del infante. Una perspectiva desde el psicoanálisis y la psicología evolutiva. Buenos Aires: Paidós, $4^{a}$ reimpresión, 2005.

Taubner, S. (2015). Konzept Mentalisieren. Eine Einführung in Forschung und Praxis. Gießen : Psychosozial-Verlag.

Wallerstein RS (1988). One psychoanalysis or many? Int J Psychoanal 69:5-21.

Wallerstein RS (1990). Psychoanalysis: The common ground. Int J Psychoanal 71:3-20.

Winnicott, D.W. (1960). Ego Distortion in Terms of True and False Self. en: Winnicott, D.W., Ed., The Maturational Processes and the Facilitating Environment: Studies in the Theory of Emotional Development, Karnac Books, London, 140-152. 
Winnicott, D.W. (1967). Mirror-role of Mother and Family in Child Development, en (1971) Playing and Reality. Routledge: London and New York, pp. 111-118.

Wolf, E.S. (1988). Theorie und Praxis der psychonalytischen Selbstpsychologie. Frankfurt am Main: Suhrkamp Verlag, 1998.

Original recibido con fecha:

Revisado:

Aceptado: $\quad 30 / 03 / 2021$ 